\title{
Correlation of Fine Needle Aspiration Cytology and Histopathology Diagnosis in the Evaluation of Breast Lumps
}

Adnan Khan,' Raza Jamali,' Muneeb Jan, ' Maria Tasneem. ${ }^{2}$

\begin{abstract}
Background: A large number of patients have been suffer from breast cancer worldwide and this trend is increasing. It is difficult to determine whether a lump is benign or malignant from clinical assessment; thus, the need for micropic and tissue analysis arises. Methods: This comparative retrospective cross sectional study was conducted in the Department of Pathology, Rehman Medical Institute, Peshawar, Pakistan from January 2006 to March 2013, to determine the value of fine needle aspiration cytology (FNAC) in the diagnosis of breast lump and to compare the result of FNAC with histological diagnosis to assess its accuracy. Results: Seventy-four cases with breast lumps were presented for FNAC. of these, $32.4 \%$ were reported as a $\mathrm{C}_{2}$ lesion, $4.1 \%$ were reported as benign with atypical cells $\left(\mathrm{C}_{3}\right), 8.1 \%$ cases were suspicious for malignancy $\left(C_{4}\right)$, and $55.4 \%$ were positive for malignancy $\left(C_{5}\right)$. On histopathology examination, out of 24 cases which were reported as $\mathrm{C}_{2}$ lesions, $95.8 \%$ were benign and $4.1 \%$ turned out to be invasive ductal carcinoma. Of the cases that presented as $\mathrm{C}_{3}$ lesions, one was diagnosed as benign duct ectasia, one with ductal carcinoma in situ, and one with invasive ductal carcinoma on histopathological examination. The cases that were diagnosed as $C_{4}$ lesions all turned out to be carcinoma on histopathology. In this study, FNAC and histopathology diagnoses were strongly correlated [r 0.92, $p<0.001]$. Conclusion: Diagnosis of breast lump based on FNAC should be practiced as there is high correlation with histopathological finding. FNAC should be used as a routine diagnostic procedure due to its cost effectiveness, thus maximizing the availability of effective health care to patients with breast lesions.
\end{abstract}

Keywords: Breast Neoplasms, biopsy, Cytological Techniques, Fine-Needle Biopsy (Source: MeSH-NLM).

\section{Introduction}

Breast cancer is the most common cancer in women both in the developed and developing world. In 2012 it were diagnosed 1.7 millions of cases worldwide and it is well-known that a half of cases and deaths occur in developing countries (International Agency for Research on Cancer, IARC. Available from: http:// www.iarc.fr/en/media-centre/pr/2013/pdfs/pr223_E.pdf, cited 2014 Jun 20).

is a th year medical student of a five year program (MBBS) at Rehman Medical College, Peshawar Pakistan.

Submission: Apr 8, 2014

Acceptance: Jun 28, 2014

Process: Peer-reviewed

Breast lumps are one of the most prevalent presenting complaints in an outpatient department (OPD) in Pakistan. ${ }^{2}$ About $90 \%$ are benign and of no grave consequences, but malignant lumps contribute to a consequential percentage of all breast lumps. ${ }^{2}$ With growing vigilance in the general population, especially about breast pathologies, and the associated solicitude and stress that this condition may lead to, the knowledge that breast cancer can have grim consequences compels patients to seek medical advice. ${ }^{3,4}$ It is difficult to determine whether a lump is benign or malignant from clinical assessment. ${ }^{5} \mathrm{~A}$ confident diagnosis can be established in more than $95 \%$ of cases utilizing triple assessment (examination, imaging and histological studies). ${ }^{6}$

Fine needle aspiration cytology (FNAC) is a relatively simple, reliable, atraumatic, economical and complication-free technique for the evaluation of mass lesions. It can be facilely reite- rated if an adequate aspirate is not obtained. ${ }^{4}$ FNAC is now a well-established technique for the investigation of women with suspected breast carcinoma.?

Fine-needle aspiration (FNA) has become one of the most important diagnostic tools for palpable breast masses and false-negative results have become a major concern. However, cytopathologists agree in certain parameters to the adequacy of an FNA specimen. ${ }^{8}$
It has been shown that FNAC has reduced the number of open biopsies because of its high diagnostic sensitivity and specificity. However, open biopsy is still preferred in some centers due to lack of expert cytologists. ${ }^{9}$ The biopsy of the palpable breast lesion predicated on the histological study of the tissue specimens can provide all the reliable information to the surgeon and oncologist for modern therapeutic strategies as part of the decision-making regarding the patient's treatment. This technique permits the eventual use of neo-adjuvant therapy. ${ }^{10}$ Different studies have determined that FNAC has a sensitivity ranging from $80 \%$ to $98 \%$ and a specificity of $99 \%$ to $100 \% .{ }^{11}$

The purpose of the present study is to determine the value of fine needle aspiration cytology in the diagnosis of breast lumps and to compare the result of FNAC with histological diagnosis to assess its accuracy.

${ }^{1}$ Rehman Medical College, Peshawar, Pakistan.

${ }^{2}$ Postgraduate trainee, Histopathology Department, Rehman Medical Institute, Peshawar, Pakistan. 


\section{Methods}

This was a comparative cross-sectional study conducted at Histopathology Department Rehman Medical Institute, Peshawar, Pakistan, from January 2006 to March 2013. Non-probability convenient sampling was used. The Ethical Review Board of Rehman Medical College, Peshawar, Pakistan approved the study. Attendants were informed of their right to refuse participation and of the respect of the confidentiality of their answers.

The guidelines of the Strengthening of the Reporting of Observational Studies in Epidemiology (STROBE) statement checklist for cross-sectional studies was followed in writing the present article. $^{12}$

All female patients with unknown primary diagnosis of breast lump undergoing FNAC followed by excision biopsy, were in cluded. Patients with recurrent malignancy, patients who underwent FNAC but did not undergo subsequent histopathological diagnosis, and patients under chemotherapeutic treatment were excluded from the study. The study included 74 cases.

FNA was performed utilizing a 5-millimeter $(\mathrm{mL})$ or $10 \mathrm{~mL}$ disposable syringe for each sample and for each patient. No local anesthetic was utilized. The needle was inserted into the palpable lesions, either once or twice depending upon the size of the nodule. Cellular material was aspirated into a syringe and expelled onto slides. Three to four slides were prepared for each patient. A small or medium-sized drop of aspirate was put near the frosted edge of a slide that was placed on a table. A second slide was acclimated to spread the aspirated material in the same manner used to prepare a peripheral blood smear and one of the smears was wet fine-tuned in $95 \%$ methanol and stained with Hematoxylin and Eosin (HCEE). The air dried smears were stained with Giemsa stain. The procedure was done within one hour, and the reports were signed-out within 2 to 3 days.

The biopsy specimens were fine-tuned in $10 \%$ formalin for 24 hours. Then gross examination was done in the Department of Pathology by consultant histopathologists. The gross and cut section findings were noted. Several bits were taken from opportune sites for processing and paraffin embedding. From each block, sections were cut at 4-5 microns thickness and stained with HCQE.

Statistical analysis was performed using the Statistical Package for Social Science (SPSS $\odot$ ) version $20 .{ }^{13}$ Continuous data was displayed as the mean \pm standard deviation (SD), while the categorical and nominal data was presented as frequencies and percentages. Pearson's correlation was applied and a p-value of $<0.05$ was taken as statistically significant with a confidence level of $95 \%$.

\section{Results}

The age range of the 74 patients included in this study was 15 70 years with mean age of $41.43 \pm 13.421$. The most effected age group was 41-50 years. Out of these 25 cases were benign and 49 were diagnosed as malignant. Highest frequencies of benign lesions were in the age group of 20-45 and the highest frequency of malignancy was in 30-55 year old women. The sensitivity of the test was $95.83 \%$ and specificity was $100 \%$. Statistical analysis by Chi-square test revealed a significant Chi-square value of $66.58(P<0.001)$ (Figure 1).

Figure 1. Age Distribution of Patients with Benign and Malignant Breast Lump.

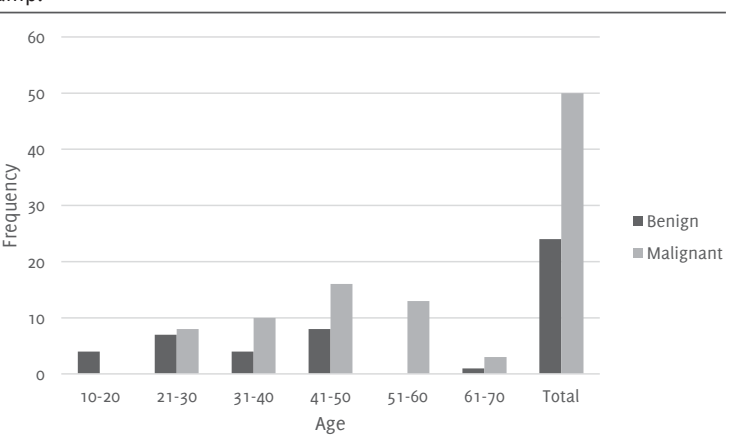

On cytology 24 patients (32.4\%) had ( $\mathrm{C}_{2}$ ) benign breast lesions. Out of these 24 cases, nine were reported as fibro-adenoma, five as fibrocystic disease, four as duct ectasia, three as proliferative breast lesion, two as lactating adenoma and one as pyogenic abscess. Out of 74 patients, three cases $(4.1 \%)$ were benign with atypical cells $\left(C_{3}\right)$, six cases $(8.1 \%)$ were suspicious for malignancy ( $\left.\mathrm{C}_{4}\right)$, and $41(55.4 \%)$ were positive for malignancy ( $\left.\mathrm{C}_{5}\right)$ (Table 1).

Out of a total of 74 cases that underwent a surgical procedure, 24 were reported as benign lesions. Among these 24 cases, eight were reported as fibro adenoma, three were diagnosed as a phylliodes tumor, five as fibrocystic disease, six as duct ectasia and one had sub-acute mastitis along with duct ectasia. Among 24 cases one was reactive hyperplasia.

The remaining 50 cases were diagnosed as carcinoma of breast on histopathology. Among these, 39 were invasive ductal carcinoma, three with invasive lobar carcinoma, and two were diagnosed as infiltrating ductal carcinoma.

One case was of mixed invasive and mucinous carcinoma, one medullary carcinoma, one with ductal carcinoma with medullary feature, one with malignant adnexal tumor, and one with border line stromal sarcoma. One was a case of in-situ ductal carcinoma. Out of these 39 cases with invasive ductal carcinoma, 21 of them were grade III, 17 grade II and only one was grade I. One case was reported as a C2 lesion (proliferative breast lesion) on biopsy and it was proved to be invasive ductal carcinoma.

Table 1. Cytology Finding of Breast Lump on Fine Needle Aspiration (FNA)

\begin{tabular}{lr}
\hline Stage $[\mathrm{n}=74]$ & Freq. $(\%)$ \\
\hline C2- Benign & $24(32.4)$ \\
\hline Fibro adenoma & $9(12.2)$ \\
\hline Fibrocystic & $5(6.8)$ \\
\hline Duct ectasia & $4(5.4)$ \\
\hline Proliferative breast lesion & $3(4.1)$ \\
\hline Lactating adenoma & $2(2.7)$ \\
\hline Pyogenic abscess & $1(1.4)$ \\
\hline C3- benign with atypical cells & $3(4.1)$ \\
\hline C4-Suspecious for malignancy & $6(8.1)$ \\
\hline C5-malignant & $41(55.4)$
\end{tabular}


Of the cases that were diagnosed as $C_{3}$ lesions (benign with atypical cells), one was diagnosed as benign duct ectasia, one with ductal carcinoma in situ and one with invasive ductal carcinoma. All six cases diagnosed as $\mathrm{C}_{4}$ lesions (suspicious for malignancy) turned out to be malignant on histopathology. Out of these, five were invasive ductal carcinoma and one was borderline stromal sarcoma. The correlation between FNAC and histopathology is shown in table 2.

\section{Discussion}

Breast cancer is one of the most frequently occurring clinical conditions worldwide. ${ }^{14}$ Although there has been little success in controlling the disease, it is of general accord that a confident preoperative diagnosis should be made before surgery, thus making FNAC a reliable diagnostic tool. It is a cheap and complication-free technique. The cost is economical, equipment is low-priced and the technique is relatively easy. ${ }^{15}$ The procedure can also be performed anywhere. Furthermore, FNAC has a high accuracy, making it a reliable procedure. ${ }^{14}$ The reesults can be obtained quickly and it is less invasive compared to tissue biopsy.

FNAC is an easily diagnostic method for determining the causes of a breast lesion. Its success is due to its accuracy and cost effectiveness for a breast lump. Therefore, it has many advantages for patients and physicians. ${ }^{16}$

In this study the sensitivity of the test was $95.83 \%$ and specificity was $100 \%$. Statistical analysis by Chi-square test revealed Chi-square value $66.58(P<0.001)$, which is statistically significant. In a similar study performed at Khyber Teaching Hospital, Peshawar, Pakistan, from August 2002 to May 2003 the sensitivity and specificity of FNAC was $91.66 \%$ and $96.96 \%$, respectively. ${ }^{3}$ the results of this study were similar to a study performed at the University Medical and Dental College, Faisalabad, Pakistan, from 2008 to 2011 which showed a sensitivity of $96.42 \%$. ' Similarly, a study performed by Waqar describes the sensitivity of FNAC as almost $92.6 \% .^{17}$ In the literature, sensitivity ranges from $94-99 \%$ and specificity reaches up to $100 \% .{ }^{11,19}$

Keeping in mind the aim and objectives of the present study, 71 of the 74 cases were diagnosed on FNAC as malignant and were correlated with their histological diagnosis. All the cytologically-diagnosed malignant lesions were proven to be malignant on histology. On FNAC, 24 were benign with one of the cases of benign breast lump turning out to be malignant on histological diagnosis. Fibro adenoma is a common benign breast lump in the majority of the young females. ${ }^{19}$ Three cases out of 74 on FNAC were $C_{3}$ lesions (benign with atypical cells) on histological finding, with one turning out to be benign and two being malignant. Six cases were diagnosed as $\mathrm{C}_{4}$ lesions (suspicious for malignancy) on FNAC with all of them being malignant on histological examination. FNAC of the breast can reduce the number of open breast biopsies. ${ }^{8}$ The procedure has some challenges due false positives or uncertain test results in the diagnosis of fibrocystic disease, adenosis, epithelial hyperplasia with or without atypia, apocrine metaplasia, radial scar, and papilloma. ${ }^{20}$

The current study showed that FNAC is a reliable method. It helps in diagnosing breast lumps without surgical intervention and it also helps in pre-operative decision-making. From this study we can presume that FNAC has an association with histopathological findings and ought to be performed on standard premises. FNAC is also a good tool for follow up if there is recurrence of breast lesions. In the presence of budget constraints and personnel shortage, hospitals are required to demonstrate even great cost effectiveness in the diagnosis of breast lesions. Confident preoperative diagnosis is becoming increasingly important. FNAC should be practiced on routine basis as it is cost effective and reliable and thus maximizes the availability of health care for patients with breast-related pathologies.

There are some difficulties and limitations that need to be mention about FNAC, first at all, both false-negative and false-positive results can occur. ${ }^{21}$ The most significant difficulty in making a diagnosis is the overlapping features of different lesions. ${ }^{22}$ Other limitations to this procedure include, for example, that the lack of cellular pleomorphism can often give a false negative diagnosis. Our study was of a retrospective design where patients not undergoing an operation were not included. This suggests the possibility that false-negative cases may have been missed.

FNAC has certain limitations on accounts of inadequate sample and suspicious diagnoses. The overlapping of cytological features can cause cytodiagnostic errors and wrong diagnoses when the histological architecture from Inflammatory lesions mimics Malignancy. ${ }^{23}$

The normal surgical protocol in Pakistan is in which the biopsy is not proceded by FNA; the FNA has not been given its due importance in Pakistan. The protocol of doing a FNA before a biopsy has been established in a few pathology labs and hospitals. This study shows how much of a useful procedure FNA can be, perhaps reinforcing the paradigm shift in modern medicine where minimal invasive or non-invasive procedures are beginning to replace invasive procedures.

Table 2. Comparison of Fine Needle Aspiration Cytology (FNAC) and Histopathology Findings.

\begin{tabular}{|c|c|c|c|}
\hline Cases (\%) & FNAC & \multicolumn{2}{|c|}{ Histopathology Finding } \\
\hline $0(0)$ & C1-unsatisfactory & 0 & 0 \\
\hline $24(32.4)$ & C2-Benign & 23 & 1 \\
\hline $3(4.1)$ & C3-Benign with atypical cells & 1 & 2 \\
\hline
\end{tabular}

Note: Fine needle aspiration cytology and histopathology diagnosis were strongly correlated, $r(72)=0.71, p<0.001$. 


\section{References}

1. Coleman MP, Quaresma M, berrino F, lutz J-M, Angelis De, capocaccia R. Cancer survival in five continents: a worldwide population-based study (CONCORD). Lancet Oncol, 2008 Aug;9(8),730-56.

2. Aslam S, Hameed S, Afzal T, Hussain A, Zafar H, Naz M, et al. Correlation of FNAC and Histological diagnosis in the evaluation of breast lumps. JUMDC. 2012 Jul-Dec;3(2):1-7.

3. Khemka A, Chakrabarti N, Shah S, Patel V. Palpable Breast Lumps: Fine-Needle Aspiration Cytology versus Histopathology: a Correlation of Diagnostic Accuracy. Internet Journal of Surgery. 2009;18(1).

4. Qureshi H, Amanullah A, Khan KM, Deeba F. Efficacy of fine needle aspiration cytology in the diagnosis of breast lumps. JPMI. 2011;21(4):301-4.

5. Yong W, Chia K, Poh W, Wong C. A comparison of trucut biopsy with fine needle aspiration cytology in the diagnosis of breast cancer. Singapore Med J. 1999 Sep;40(9):587-9.

6. Rahman S, Ali M, Khalili Y, Ali A, Lal A. Role of fine needle asporation cytology in evaluating the breast lumps. Comal J Med Sci. 2011 Jan-Jun;9(1):59-61. 7. Baak J.P.A. The relative prognostic significance of nucleolar morphology in invasive ductal breast cancer. Histopathology. 1985 Apr;9(4):437-44.

8. Boerner $S$, Sneige $N$. Specimen adequacy and false-negative diagnosis rate in fine-needle aspirates of palpable breast masses. Cancer. 1998 Dec $25 ; 84(6): 344-8$..

9. Feichter GE, Haberthür F, Gobat S, Dalquen P. Breast cytology. Acta cytologica. 1997 Mar-Apr;41(2):327-32.

10. Bajwa R, Zulfiqar T. Association of fine needle aspiration cytology with tumor size in palpable breast lesions. Biomedica. 2010 Jul-Dec;26:124-9.

11. Wilkinson EJ, Bland KI. Techniques and results of aspiration cytology for diagnosis of benign and malignant diseases of the breast. Surg Clin North Am. 1990 Aug;70(4):801-13.

12. von Elm E, Altman DG, Egger M, Pocock SJ, G $\phi$ tzsche PC, Vandenbroucke JP; STROBE Initiative. The Strengthening the Reporting of Observational Stu- dies in Epidemiology (STROBE) statement: guidelines for reporting observational studies. PLOS Med. 2007 Oct 16;4(10):e296.

13. IBM Corp. Released 2011. IBM SPSS Statistics for Windows, Version 20.0 Armonk, NY: IBM Corp.

14. Sohail S, Alam SN. Breast cancer in pakistan-awareness and early detection. Journal of the College of Physicians and Surgeons--Pakistan: JJ Coll Physicians Surg Pak. 2007 Dec;17(12):711-2.

15. Nasuti JF, Gupta PK, Baloch ZW. Diagnostic value and cost-effectiveness of on-site evaluation of fine-needle aspiration specimens: review of 5,688 cases. Diagn Cytopathol. 2002 Jul;27(1):1-4.

16. Rupom TU, Choudhury T, Banu SG. Study of Fine Needle Aspiration Cytology of Breast Lump: Correlation of Cytologically Malignant Cases with Their Histological Findings. BSMMU J. 2011;4(2):60-64.

17. Waqar AJ, Zada N and Israr M. Comparison of FNAC and core needle biopsy for evaluating breast lumps. JCPSP. 2002; 12: 686-8.

18. Gukas I, Nwana E, Ihezue C, Momoh J, Obekpa P. Tru-cut biopsy of palpable breast lesions: a practical option for pre-operative diagnosis in developing countries. Cent Afr J Med. 2000 May;46(5):127-30.

19. Tiryaki T, Senel E, Hucumenoglu S, Cakir BC, Kibar AE. Breast fibroadenoma in female adolescents. Saudi Med J. 2007 Jan;28(1):137-8.

20. Guray M, Sahin AA. Benign breast diseases: classification, diagnosis, and management. Oncologist. 2006 May;11(5):435-49.

21. Bukhari MH, Arshad M, Jamal S, Niazi S, Bashir S, Bakhshi IM. Use of fine-needle aspiration in the evaluation of breast lumps. Patholog Res Int. 2011;2011:689521.

22. Bakhos R, Selvaggi SM, Dejong S, Gordon DL, Pitale SU, Herrmann M, Wojcik EM. Fine-needle aspiration of the thyroid: rate and causes of cytohistopathologic discordance. Diagn Cytopathol. 2000 0ct;23(4):233-7.

23. Bagga PK, Mahajan NC. Fine needle aspiration cytology of thyroid swellings: how useful and accurate is it?. Indian J Cancer. 2010 0ct-Dec;47(4):437-42.

\section{Acknowledgments}

Prof. Ashraf Qamar, Histopathology Department, Rehman Medical Institute, Peshawar, and Dr Iftikhar Qayum, Research Department, Rehman Medical College, Peshawar (KPK) are acknowledged for support and advice.

\section{Conflict of Interest Statement a Funding}

The Authors have no funding, financial relationships or conflicts of interest to disclose.

\section{Author Contributions}

Conception and design the work/idea: AK. Collect data/obtaining results: AK RZ MJ MT. Analysis and interpretation of data: AK RZ. Write the manuscript: AK RZ MJ MT. Critical revision of the manuscript: AK RZ MJ MT. Approval of the final version: AK. Contribution of patients or study material: AK. Statistical advice: AK RZ. Administrative or technical advice: AK MJ MT.

\section{Cite as:}

Khan A, Jamali R, Jan M, Tasneem M. Correlation of Fine Needle Aspiration Cytology and Histopathology Diagnosis in the Evaluation of Breast Lumps. Int J Med Students. 2014 Mar-Jun;2(2):40-3. 